Corresponding Author:

Rizky Nurdiansyah

rizky.nurdiansyah@i3l.ac.id

Received: 1 February 2020

Accepted: 8 February 2020

Published: 16 February 2020

Publishing services provided by

Knowledge E

(c) Kevin Nathanael Ramanto

et al. This article is distributed

under the terms of the

Commons Attribution License,

which permits unrestricted use and redistribution provided that the original author and source are credited.

Selection and Peer-review under the responsibility of the IC-BIOLIS Conference Committee.

\section{The Evolution Study Of 6-Cysteine Family Member Protein of Plasmodium sp. As a Potential Drug Candidate Against Malaria Infection}

\section{Kevin Nathanael Ramanto1, Rizky Nurdiansyah¹, and Priscilla Jessica²}

${ }^{1}$ Department of Bioinformatics, School of Life Sciences, Indonesia International Institute for Life Sciences, Jakarta 13210 Indonesia

${ }^{2}$ Department of Biotechnology, School of Life Sciences, Indonesia International Institute for Life Sciences, Jakarta 13210 Indonesia

\section{Abstract}

The increase of current antimalarial drug resistance was reported and lead to the greatest threats to malaria control; therefore, new methods should be applied to encounter this problem. Although the protein evolution study of Plasmodium may contain valuable information in finding a new antimalarial drug candidate, the cross-species antimalaria drug cannot be made because there is no sufficient information regarding the protein evolution between human-infecting and noninfectious Plasmodium. In this study, data mining from PlasmoDB discovers several proteins shared by Plasmodium where some of them include in a 6-Cysteine protein family. Previous studies revealed that 3 of 6 -Cysteine family members (P41, P48/45, and P230) could be used as a vaccine candidate. From this information, the evolution properties and the characteristics of these proteins were further analyzed. Protein sequences of 6-Cysteine protein family members were retrieved from plasmoDB and the GenBank. Maximum likelihood phylogenetic tree and time trees were then constructed by using MEGAX, protein domain analysis was done by using InterPro, and all tertiary structures of these proteins were predicted by using PHYRE2. Phylogenetic tree and time tree analysis showed that the human-infecting and the non-infectious Plasmodium have a different cluster and evolutionary rates. Furthermore, several domains that can be used vaccine targets were found in P41, P48/45, and P230, such as transmembrane, signal peptide, and a coiled-coil domain. Tertiary structure prediction also revealed a different characteristic of these proteins. Thus, our findings provide valuable information to support the development of the cross-species antimalaria vaccine using 6-Cysteine protein family members.

Keywords: 6-Cysteine, drug target, protein domain, protein evolution, tertiary structure, Plasmodium

\section{Introduction}

Malaria is one of the endemic diseases that occur in developing countries, including Indonesia. According to the world malaria report 2018, there are 219 million cases of malaria with 435000 deaths worldwide [1]. On the other hand, the Indonesian 
government estimated about 10 to 12 million people had suffered malaria, and 30000 of them died each year [2]. From 34 province of Indonesia, Papua has the highest prevalence of malaria $(12,07 \%)$ indicated that malaria infection still become one of the greatest threats towards human health in Indonesia [2].

A parasite called plasmodium $s p$. is responsible for the cause of malaria throughout many years. Plasmodium spp. have a complex life cycle alternating between female Anopheles mosquitoes and vertebrate hosts, which require the formation of unique zoite forms to invade different cell types [3]. When sporozoites enter the host, they will infect hepatocytes, followed by the asexual cycle in the blood [3]. Furthermore, sexual forms that develop during the blood-stage are ingested by a feeding mosquito and completing the cycle. Currently, there are five plasmodial species consider as health threats for humans, which are: P. falciparum, vivax, ovale, knowlesi, and malariae [3].

Artemisinin Combination Treatment (ACT) is one of the treatments for malaria that are commonly used in Indonesia [2]. The ACT is a combination of antimalaria drug artemisinin group with other antimalaria groups. Artemisinin act as a shortacting drug for rapid parasitemia reduction while the second antimalaria group serves as a longer-acting partner, which eliminates parasite that survives artemisinin [4]. Currently, there are three types of ACT that available in Indonesia, such as (i) Dihydroartemisinin+Piperaquine; (ii) Artesunate+Amodiaquine; (iii) and Artemether + Lumefantrine [2]. The duration of the ACT for each malaria patient depends on the cause of the malaria infection experienced by the patient [2]. However, the emergence and spread of ACT resistance lead to the most significant threats in malaria control [5].

Previous studies were demonstrated ACT resistance developed by plasmodial species. In 2018, Bakhiet et al. observed the evolution of known drug resistance markers in Plasmodium falciparum that lead to ACT failure [6]. In this study, Bakhiet et al. genotyped known drug resistance markers (Pfcrt, Pfmdr-1, Pfdhfr, Pfdhps, Pfk13 propeller) and their flanking microsatellite among Plasmodium falciparum which obtained between 2009 to 2016 in different regions in Sudan and data were compared with published findings pre-ACT [6]. The result showed a high prevalence of Pfcrt76T, Pfmdr-1-86Y, Pfdhfr51l, Pfdhfr108N, Pfdhps37G in all regions of Sudan, which indicate drug resistance genes in Sudan correlate with the drug deployment pattern [6]. Thus, a new method of treatment should be considered to encounter the spread of ACT resistance.

In this study, several proteins shared by plasmodium were found by data mining from PlasmoDB where some of them include in 6-Cysteine (6-Cys) protein family which expressed in different stages throughout the parasitic cycle of plasmodium [7]. Previous studies revealed that 3 of the 6 -Cys family member could be used as vaccine target 
candidate, which are: (i) P41 which has a signal sequence and located on the surface of the merozoite; (ii) P48/45 which involved in male or female gamete fusion in the mosquito midgut; (iii) and P230 that also associated with the gamete membrane by binding to P48/45 [7], [8].

Previous evolution study of each of these proteins contain valuable information regarding the characteristic of 6-Cys family member protein and support the development of malaria vaccine. The first study of polymorphism and natural selection of P41 was done by Ahmed et al. where they discover a low level of polymorphism in both s48/45 domains in P41 from P. falciparum, vivax, and knowlesi [9]. In 2018, Srisutham et al. also showed that P48/45 in P. malariae has low average pairwise nucleotide diversity and haplotype diversity compared to thrombospondin-related anonymous protein (TRAP) and apical membrane antigen 1 (AMA1) [10]. Another research conducted by MacDonald et al. discovers that recombinant P230 domain 1 in P. falciparum has a similar secondary structure after heating to denaturation levels and cooling which indicates P230 has suitable thermal stability for vaccine component [11]. All of these studies have revealed the characteristic of $\mathrm{P} 41, \mathrm{P} 48 / 45$, and $\mathrm{P} 230$ in Plasmodium sp. could be used as a vaccine target candidate in the future. However, the cross-species antimalaria vaccine cannot be made because there is no sufficient information regarding the protein evolution between human-infecting and non-infectious Plasmodium.

Vaccines are the most reliable alternative method for malaria control but their development has been limited by strain specificity in previously studied antigens which makes the proposed vaccine is ineffective against all five human-infecting Plasmodium. Furthermore, the specific characteristic of these studied antigens in human infecting Plasmodium has remained unknown. It is important to elucidate the differences between human infecting and non-human infecting Plasmodium of studied antigens to determine which antigens that effectively elicit immunity against malaria. Since the 6-Cys protein family members presence in all Plasmodium species, then the cross-species antimalaria vaccine could be made by using P41, P48/45, and P230 [7]. The objective of this study is to provide valuable information regarding the evolutionary relationship of $\mathrm{P} 41, \mathrm{P} 48 / 45$, and P230 by implementing the phylogenetic analysis. Furthermore, the characteristic differences of these proteins in each plasmodium were observed in $2 D$ and $3 D$ protein structure. Taken together, these results provide valuable information as well as support the development of the malaria vaccine using 6-Cys family member protein. 


\section{Methods}

Eight protein sequences of each P41 and P230 were retrieved from PlasmoDB (https: //plasmodb.org/plasmo/) while another eight protein sequences of P48/45 were taken from the GenBank (https://www.ncbi.nlm.nih.gov); detail is shown in table 1 [12]. From eight plasmodium, five of them could infect humans (P. falciparum, P. vivax, P. knowlesi, $P$. ovale, and P. malariae) and the other (P. berghei, P. chabaudi, and P. reichenowi) could not infect human but could infect other species. Multiple sequence alignment was done by using the MUSCLE algorithm [13]. Finding the best maximum likelihood model for each protein sample, phylogenetic tree analysis, and time tree analysis was conducted with MEGAX software [14]. All phylogenetic tree was constructed by using Jones, Taylor, and Thornton with gamma distribution and frequencies model ( $J T T+G+F$ ) [15]. One thousand replication bootstraps were performed to test each of the tree robustness. The time tree was constructed by following Mello's protocol with the default parameter from MEGAX software [16]. Time tree calibration was retrieved from the time tree database (http://timetree.org/) [17]. Domain analysis and 3D structure prediction were done by using InterPro (https://www.ebi.ac.uk/interpro/beta/) and PHYRE2 fold recognition server (http://www.sbg.bio.ic.ac.uk/phyre2/html/page.cgi?id=index) [18], [19]. Each of the protein structures was visualized by using PyMol 2.3 [20].

TABLE 1: Protein sequence detail information.

\begin{tabular}{|c|c|c|c|c|c|}
\hline \multicolumn{2}{|c|}{ P41 } & \multicolumn{2}{|c|}{ P48/45 } & \multicolumn{2}{|c|}{ P230 } \\
\hline Gene ID & Species & $\begin{array}{c}\text { Accession } \\
\text { Number }\end{array}$ & Species & Gene ID & Species \\
\hline $\begin{array}{l}\text { PF3D7_- } \\
0404900\end{array}$ & $\begin{array}{c}\text { P. falciparum } \\
\text { 3D7 }\end{array}$ & SBS87718 & P. malariae & $\begin{array}{r}\text { PBANKA_ } \\
0306100\end{array}$ & $\begin{array}{l}\text { P. berghei } \\
\text { ANKA }\end{array}$ \\
\hline $\begin{array}{l}\mathrm{PKNH}_{-} \\
0303000\end{array}$ & $\begin{array}{l}\text { P. knowlesi } \\
\text { strain } \mathrm{H}\end{array}$ & SCM16869 & P. berghei & $\begin{array}{l}\text { PF3D7_- } \\
0209000\end{array}$ & $\begin{array}{l}\text { P. falciparum } \\
\text { 3D7 }\end{array}$ \\
\hline $\begin{array}{l}\text { PVP01_- } \\
0304300\end{array}$ & $P$. vivax $\mathrm{P01}$ & SBS96099 & P. ovale curtisi & $\begin{array}{l}\mathrm{PKNH}_{-} \\
0412100\end{array}$ & $\begin{array}{l}\text { P. knowlesi } \\
\text { strain } \mathrm{H}\end{array}$ \\
\hline $\begin{array}{l}\text { PBANKA_- } \\
1002600\end{array}$ & $\begin{array}{c}\text { P. berghei } \\
\text { ANKA } \\
\text { (putative) }\end{array}$ & SBT40052 & $\begin{array}{l}\text { P. ovale } \\
\text { wallikeri }\end{array}$ & $\begin{array}{l}\text { PmUG01_- } \\
04023100\end{array}$ & $\begin{array}{c}\text { P. malariae } \\
\text { UG01 (putative) }\end{array}$ \\
\hline $\begin{array}{l}\text { PmUG01_- } \\
03015000\end{array}$ & $\begin{array}{c}\text { P. malariae } \\
\text { UG01 (putative) }\end{array}$ & OTN66276 & P. knowlesi & $\begin{array}{l}\text { PocGH01_- } \\
04021000\end{array}$ & $\begin{array}{l}\text { P. ovale curtisi } \\
\text { GH01 (putative) }\end{array}$ \\
\hline $\begin{array}{l}\text { PocGH01_- } \\
03012400\end{array}$ & $\begin{array}{l}\text { P. ovale curtisi } \\
\text { GH01 (putative) }\end{array}$ & SCM18667 & P. berghei & $\begin{array}{l}\text { PVP01_- } \\
0415800\end{array}$ & $\begin{array}{l}\text { P. vivax P01 } \\
\text { (putative) }\end{array}$ \\
\hline $\begin{array}{l}\text { PCHAS_- } \\
1003500\end{array}$ & $\begin{array}{l}\text { P. chabaudi } \\
\text { (putative) }\end{array}$ & SCM13221 & $\begin{array}{l}\text { P. chabaudi } \\
\text { adami }\end{array}$ & $\begin{array}{l}\text { PCHAS }_{-} \\
0308300\end{array}$ & P. chabaudi \\
\hline $\begin{array}{l}\text { PRDC_- } \\
0402500\end{array}$ & $\begin{array}{c}\text { P. reichenowi } \\
\text { (putative) }\end{array}$ & CDO66251 & P.reichenowi & $\begin{array}{l}\text { PRCDC_- } \\
0207900\end{array}$ & P. reichenowi \\
\hline
\end{tabular}




\section{Results and Discussions}

\section{1. $\mathrm{P} 41$}

The phylogenetic analysis of P41 protein showed two different clades between human infecting and non-human infecting plasmodium (Figure 1). As shown in the phylogenetic tree (Figure 1) and time tree analysis (Figure 2), P41 protein of $P$. vivax and $P$. knowlesi have a close relationship with each other. This result is supported by the fact that both of these parasites is originated from the malarial parasite of non-human primates [21]. In 2007, Cornejo and Esalante proved this theory by analyzing the phylogeny of complete mitochondrial genomes data of $P$. vivax and non-human primates in Southeast Asia [21].

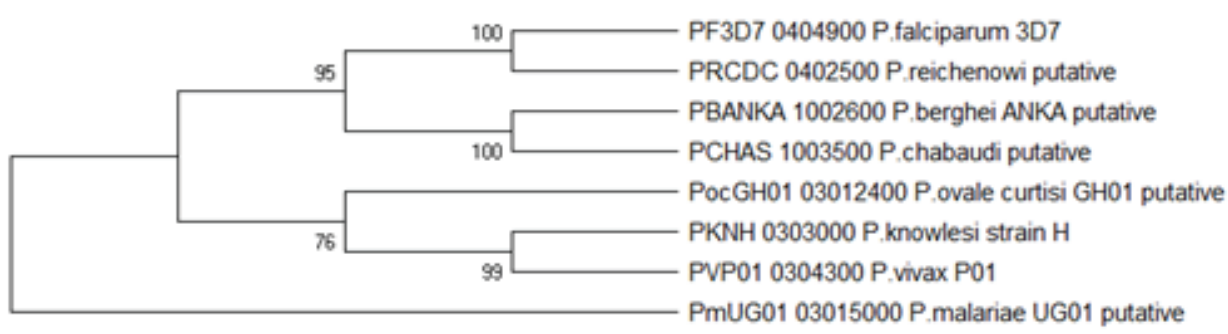

Figure 1: Phylogenetic analysis of $\mathrm{P} 41$ constructed by using the $\mathrm{JTT}+\mathrm{G}+\mathrm{F}$ model with 1000 bootstrap.

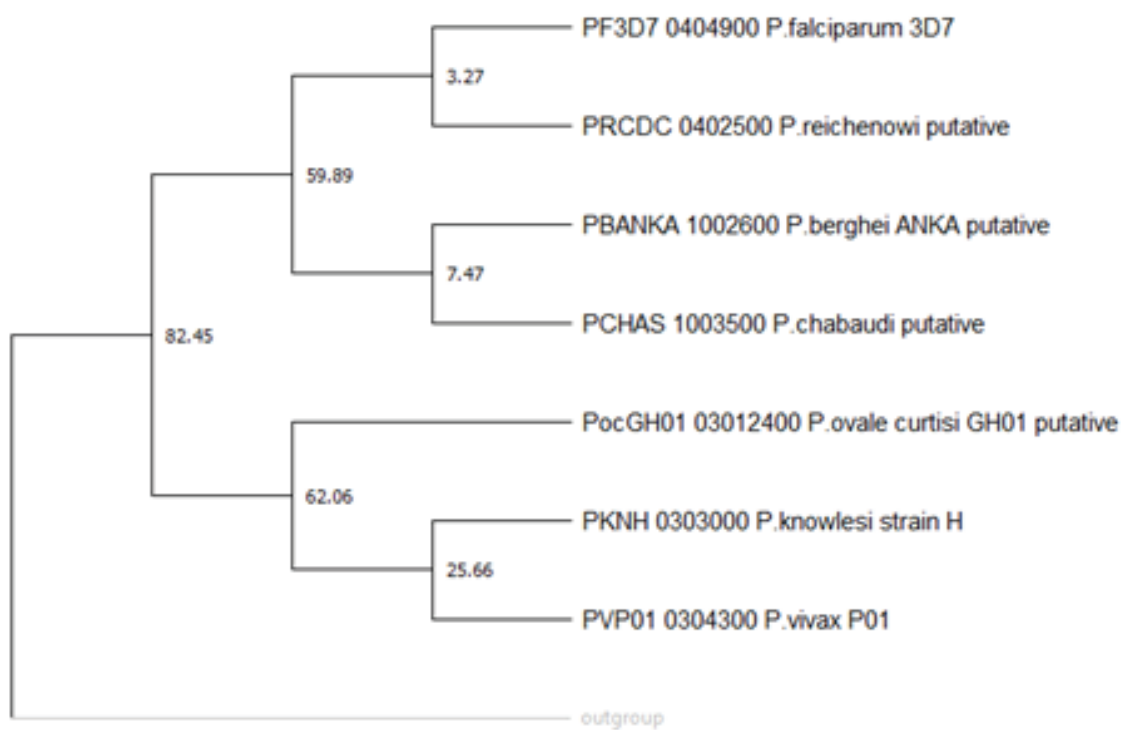

Figure 2: Time tree analysis of P41.

Another impressive result found in non-human infecting Plasmodium clade, where $P$. falciparum that initially known as human infecting Plasmodium was included in nonhuman infecting Plasmodium clade (Figure 1). Furthermore, $P$. falciparum has a close relationship with $P$. reichenowi, which only affects chimpanzee; as shown in the time 
tree (Figure 2). This result may indicate that the P41 protein of $P$. falciparum has similar characteristics with non-human infecting Plasmodium. The previous study conducted by Prugnolle et al. found the same result by exploring the diversity of Plasmodium species infecting monkeys in Central Africa [22]. Their findings suggest that $P$. falciparum can switch host between non-human primates and humans [22].

From eight Plasmodium species, P. malariae has the farthest relationship (Figure 2) and does not include in either non-human infecting and human infecting Plasmodium clade (Figure 1). This may show that P41 protein from P. malariae has different characteristics compared to other human infecting Plasmodium. Therefore, MEGAX software was recognized $P$. malariae as the outgroup among $P 41$ protein sequences [14].

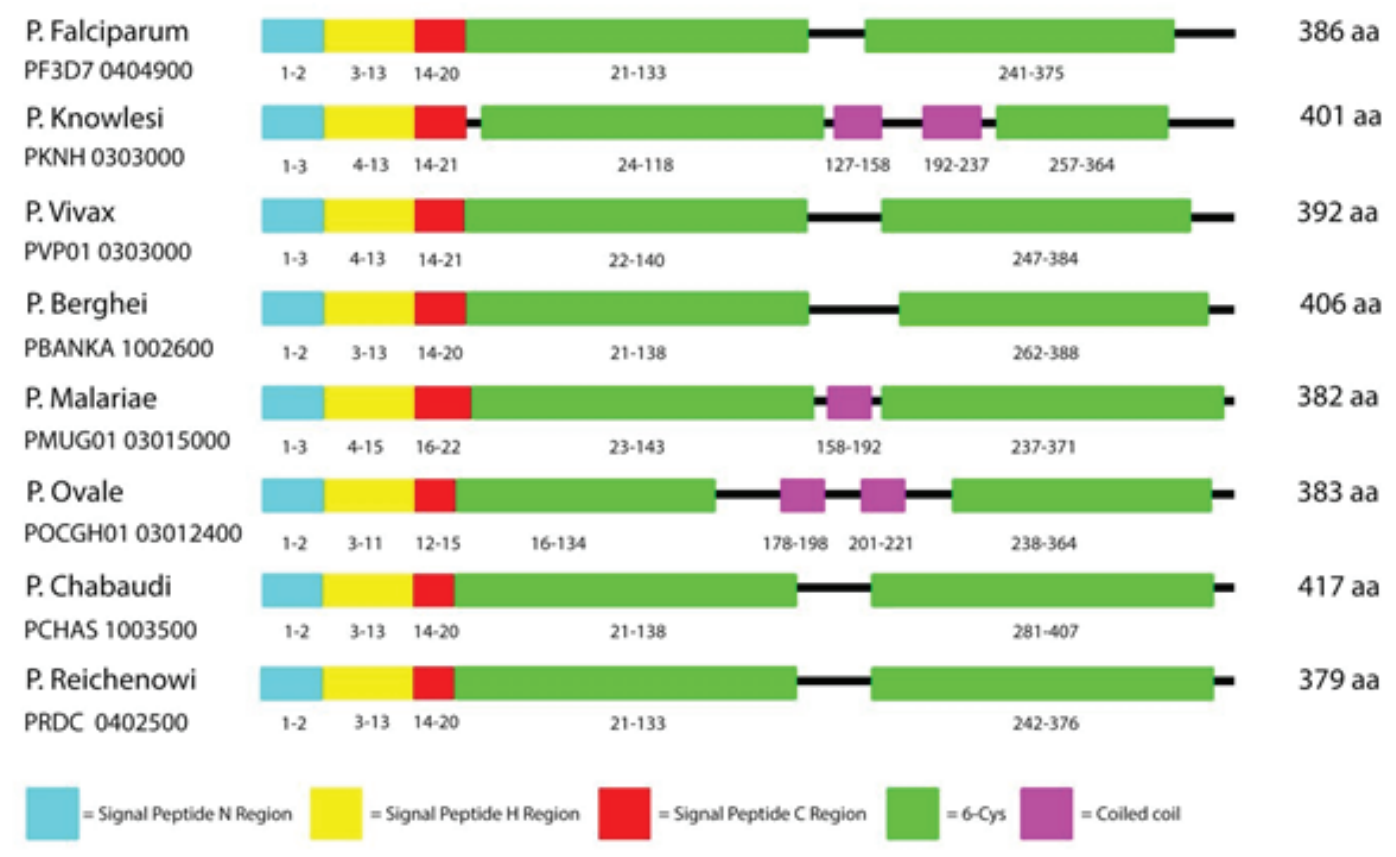

Figure 3: Domain prediction of P41protein by using InterPro.

Domain analysis detects the presence of signal peptide and coiled-coil region that could be used as a vaccine target [23], [24]. Signal peptide region present in all plasmodial species while the coiled-coil region only present in P. knowlesi, $P$. malariae, $P$. ovale. However, $P$. malariae has only one coiled-coil region compared to $P$. knowlesi and $P$. ovale that have two coiled-coil regions (Figure 3 ), which indicate the unique characteristic of $\mathrm{P} 41$ protein from $P$. malariae. The location of the signal peptide region and the coiled-coil region was described in Figure 3 where signal peptide present at the beginning of $\mathrm{P} 41$ protein sequences and coiled-coil present between two 6-Cys regions. P. knowlesi has a unique domain distribution where a small gap presents between signal peptide c-region and 6-Cys regions compared to other Plasmodium species. 
Signal peptide consists of three main parts, which are: $\mathrm{n}$-region, h-region, and cregion, which responsible for targeting proteins to the endomembrane system [23]. Targeting protein with a signal peptide region as a drug or vaccine candidate might influence protein targeting to various extracellular or subcellular compartments in the Plasmodial species [23]. On the other hand, the coiled-coil region known to induce the immune response and block critical functions in multiple pathogens, including plasmodium [24].

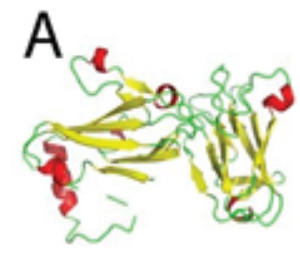

$\mathrm{E}$

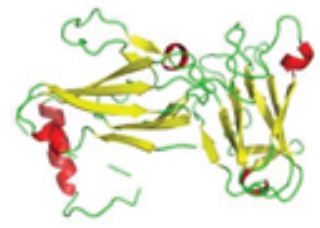

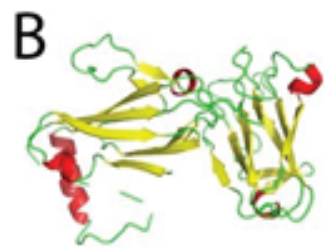

$\mathrm{F}$

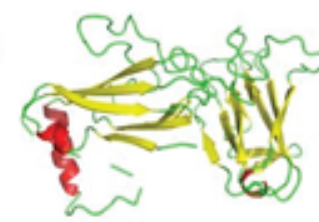

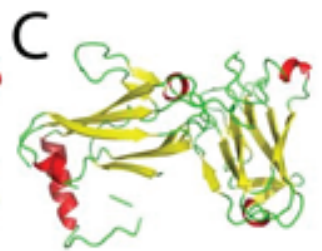

G

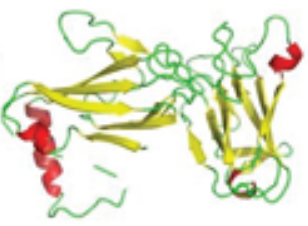

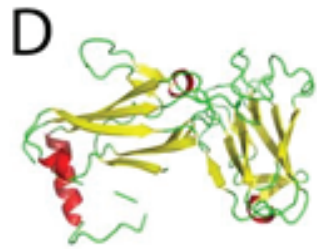

$\mathrm{H}$

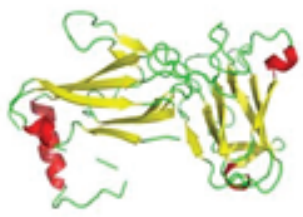

Figure 4: 3D protein structure of P41 protein predicted by using PHYRE2 and visualized by using PyMol version 2.3: (A) P. berghei, (B) P. chabaudi (C) P. reichenowi, (D) P. falciparum, (E) P. vivax, (F) P. knowlesi, (G) P. ovale, and (H) P. malariae.

In the 3D protein structure, several differences can be spotted between plasmodial species (Figure 4). For example, the present and the location of the small alphahelix structure is different in each plasmodial species (Figure 4). However, there is no significant difference between human infecting, and non-human infecting plasmodium was spotted. Thus, P41 could be used as a cross-species vaccine for malaria infection.

\section{2. $P 48 / 45$}

Phylogenetic analysis of P48/45 showed that $P$. malariae and $P$. knowlesi include in nonhuman infecting Plasmodium clade where $P$. ovale species stands as an outgroup. This result indicates $\mathrm{P} 48 / 45$ protein in $P$. malariae and $P$. knowlesi have a close relationship with non-human infecting Plasmodium (Figure 5). Furthermore, $P$. malariae and $P$. knowlesi have a homolog relationship with each other, which indicates the P48/45 in $P$. malariae and $P$. knowlesi have similar characteristics. These findings were supported by the fact that $P$. malariae and $P$. knowlesi are morphologically similar, which firstly mentioned by Coatney et al., back in 1971 [25]. 


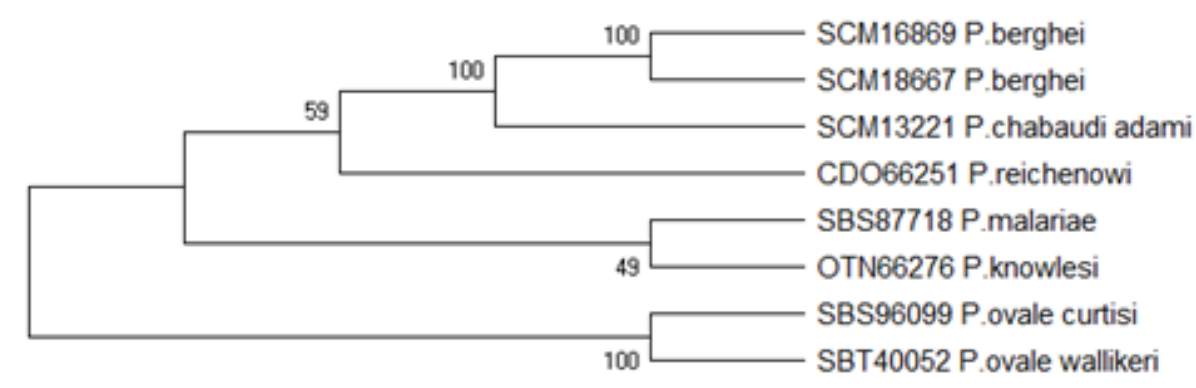

Figure 5: Phylogenetic analysis of $\mathrm{P} 48 / 45$ constructed by using the $\mathrm{JTT}+\mathrm{G}+\mathrm{F}$ model with 1000 bootstrap.

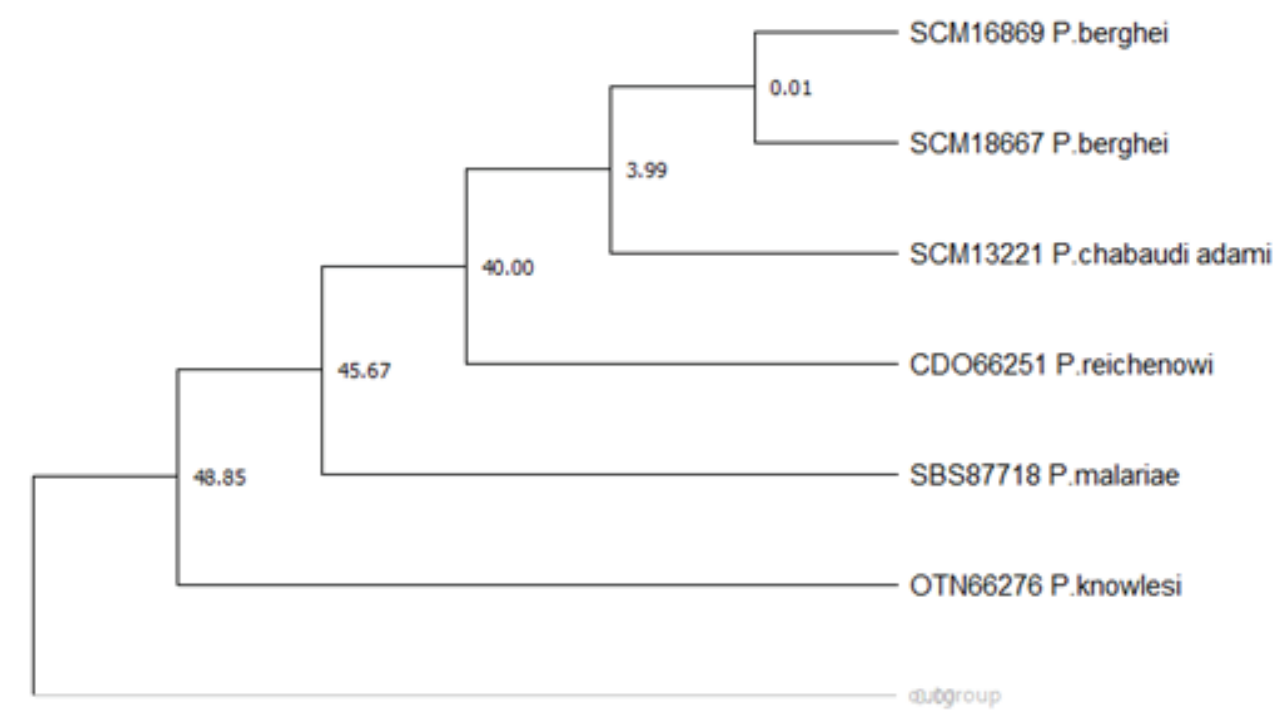

Figure 6: Time tree analysis of P48/45.

However, there is no homolog relationship between Plasmodium species found in the time tree analysis where human infecting Plasmodium have a distant relationship with non-human infecting Plasmodium (Figure 6). The result of the time tree analysis may suggest that each of the P48/45 protein in Plasmodium species have a unique characteristic. Time tree analysis also showed the farthest evolutionary rate of P48/45 protein from both $P$. ovale species compared to other Plasmodium species (Figure 6).

Compared to $\mathrm{P} 41$ domain distribution (Figure 3), the signal peptide region in P48/45 protein was only found in several Plasmodium species and with more extended size, such as P. malariae, P. ovale curtisi, P. knowlesi, P. chabaudi and, P. reichenowi (Figure 7). Furthermore, all of the P48/45 protein in all Plasmodium species contain a transmembrane region were mostly present at the end of $\mathrm{P} 48 / 45$ protein sequences which could be used as a vaccine target [26]. The different distribution of the transmembrane 


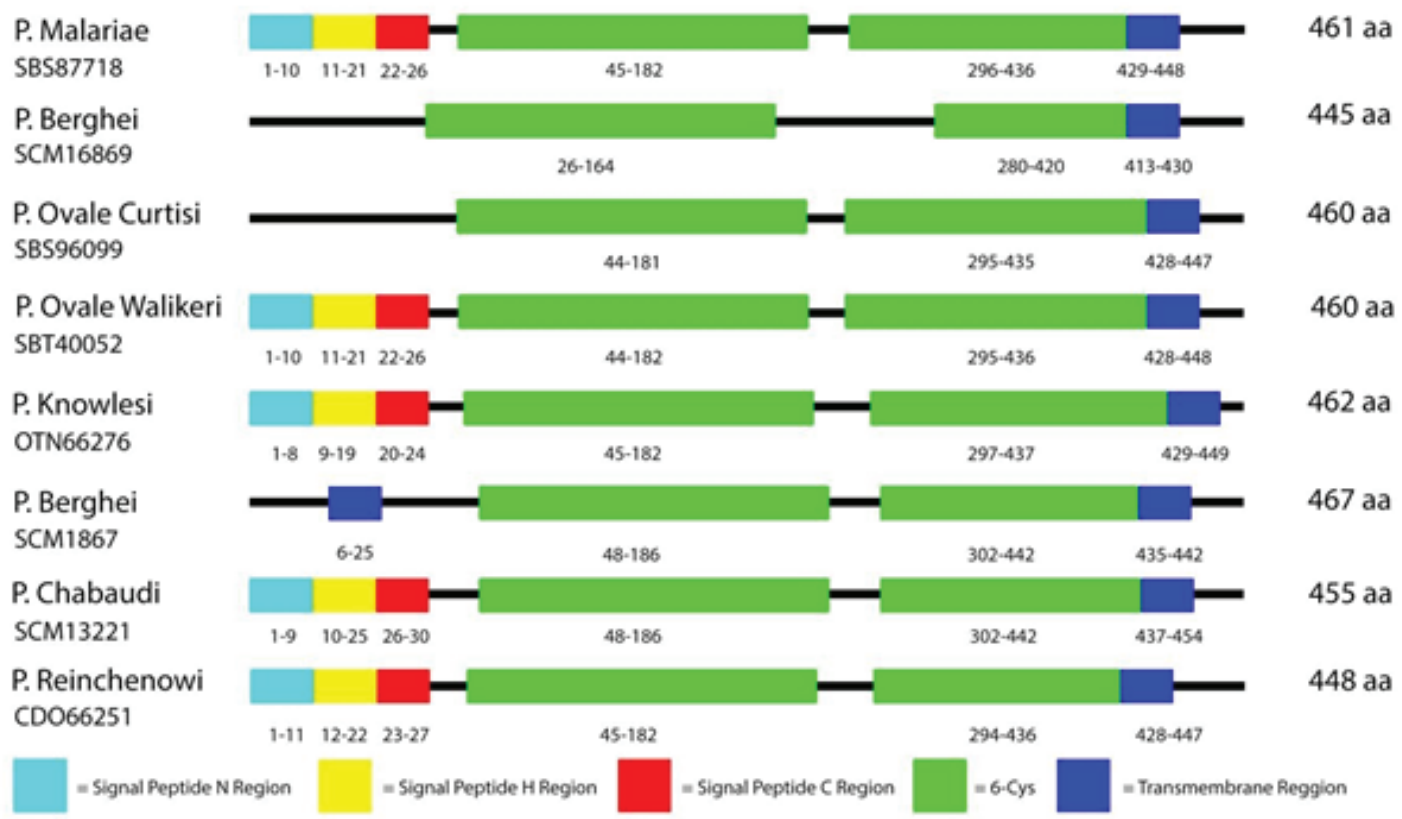

Figure 7: Domain prediction of P48/45 protein by using InterPro

region was observed in one of the P. berghei with accession number SCM 1687, where there are two transmembrane regions present in the sequences (Figure 7). Since the transmembrane region previously is known to have high CD8 $+\mathrm{T}$ cell epitope densities and broadly immunogenic, then targeting a transmembrane region in P48/45 protein in Plasmodium species can induce the maximal immune activation [26]. Therefore, these findings may suggest that $\mathrm{P} 48 / 45$ protein is suitable for a malaria vaccine.

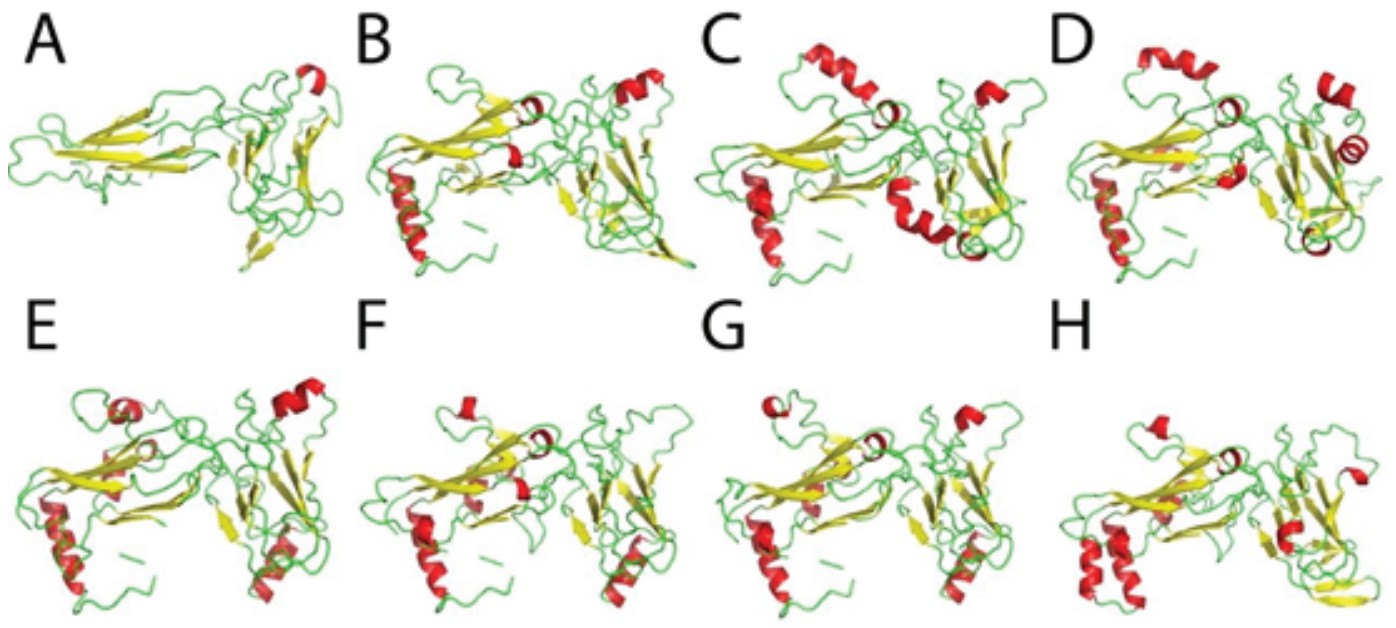

Figure 8: 3D protein structure of P48/45 protein predicted by using PHYRE2 and visualized by using PyMol version 2.3: (A) P. berghei, (B) P. berghei, (C) P. chabaudi, (D) P. reichenowi, (E) P. knowlesi, (F) P. Ovale curtisi, (G) P. Ovale walikeri, and (H) P. malariae. 
Similar to P41 protein structure prediction (Figure 4), there are several differences observed in each Plasmodium species, such as the distribution of alpha-helix and beta-sheet (Figure 8). The unique protein structure was observed in $P$. berghei with accession number SCM 16869, where only one alpha-helix was found inside the protein structure (Figure 8A). However, there are no significant differences found between human infecting and non-human infecting Plasmodium. This result may indicate that P48/45 protein may be suitable for cross-species vaccine target similar to P41 protein.

\section{3. $\mathrm{P} 230$}

The phylogenetic analysis of P230 protein showed two different clades Plasmodium (Figure 9) where $P$. knowlesi and $P$. vivax were identified as the outgroup. P. falciparum and $P$. reinchenowi have a homolog relationship with a close evolutionary rate similar to P41 protein (Figure 9 and Figure 10). Not only that, $P$. knowlesi and $P$. vivax also have a homolog relationship with the distant evolutionary rate with other Plasmodium species (Figure 9 and Figure 10).

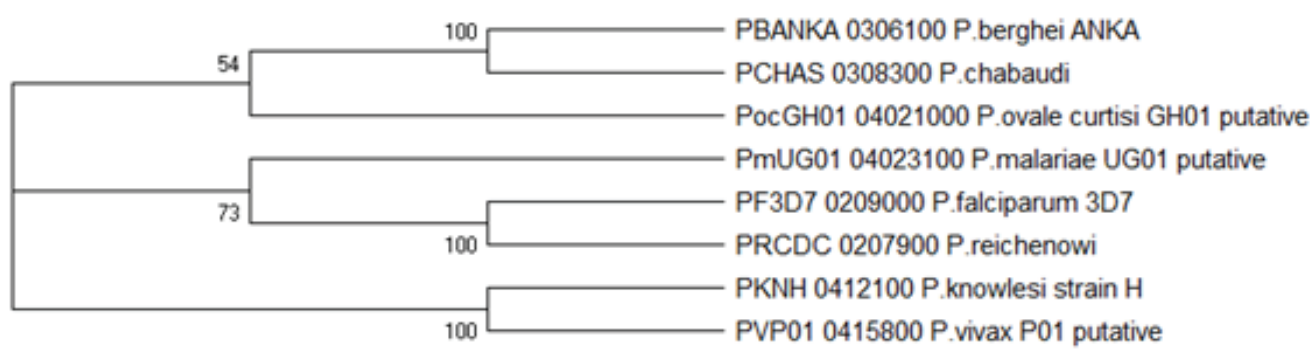

Figure 9: Phylogenetic analysis of $\mathrm{P} 230$ constructed by using the $\mathrm{JTT}+\mathrm{G}+\mathrm{F}$ model with 1000 bootstrap.

The unique relationship of $P$. malariae was observed in the time tree analysis where $P$. malariae stands alone in the time tree with the distant evolutionary rate as the outgroup (Figure 10). This finding may suggest there is a unique characteristic of P230 protein from P. malariae compared to other human infecting Plasmodium. The ortholog relationship was observed between $P$. ovale and rodent infecting Plasmodium ( $P$. berghei and $P$. chabaudi).

The domain analysis showed that all Plasmodium species have a signal peptide region and some of them ( $P$. berghei, $P$. malariae, and $P$. vivax) have a coiled-coil region. Both of these domains could be used as a vaccine target similar to P41 protein [23], [24]. However, the coiled-coil region that was detected in P230 protein is smaller compared to P41 protein. The bigger size of the coiled-coil region was only discovered 


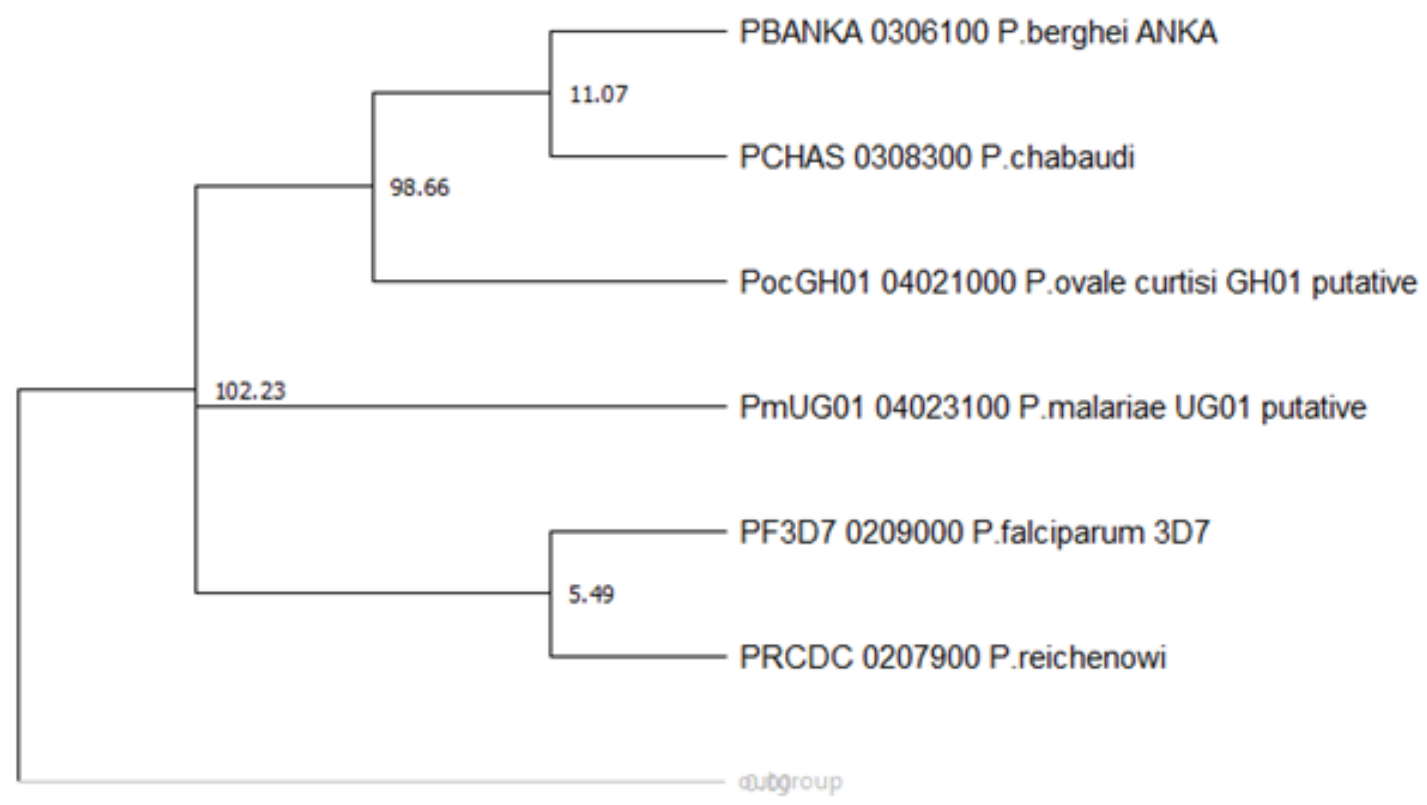

Figure 10: Time tree analysis of P230.

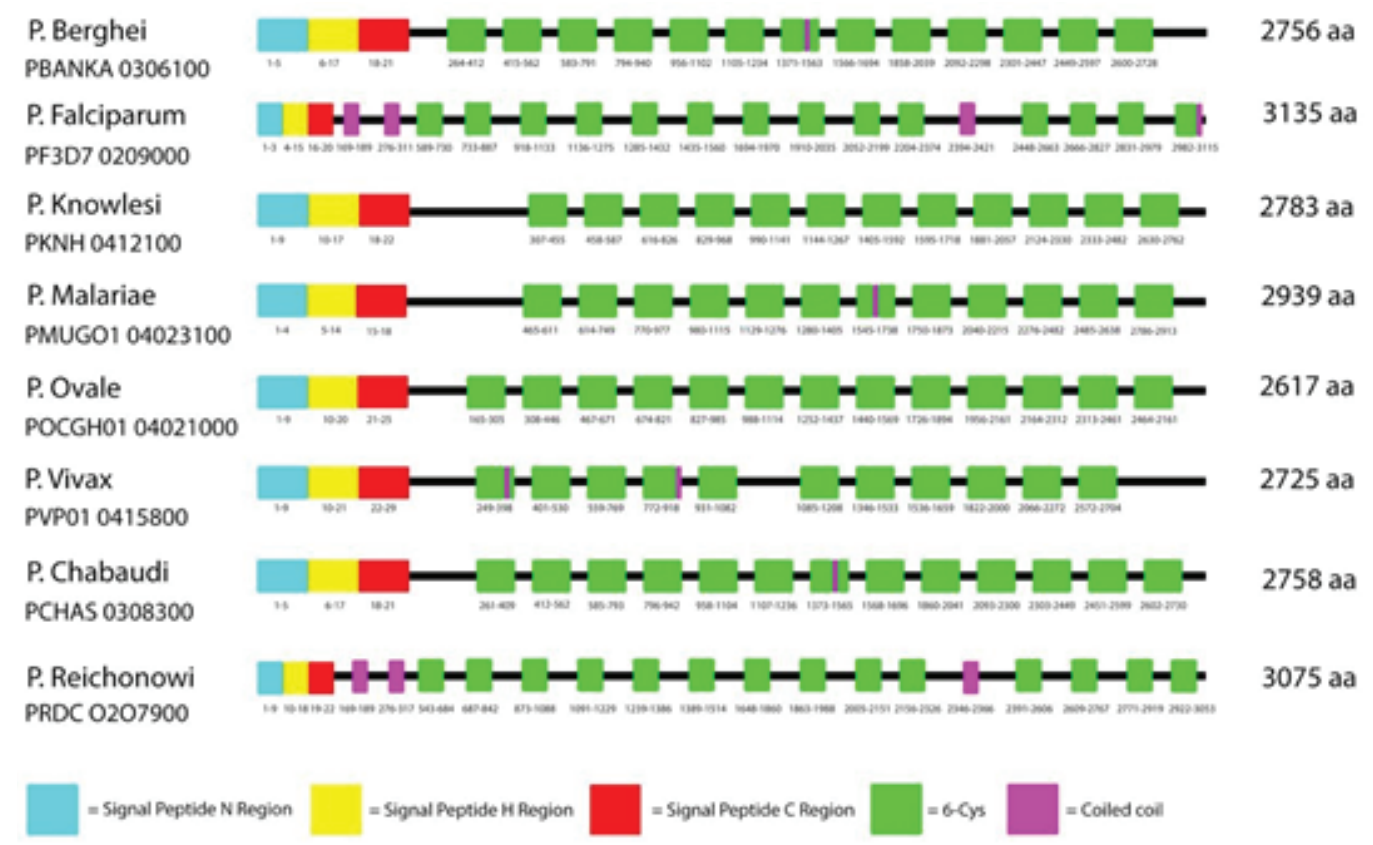

Figure 11: Domain prediction of P230 protein by using InterPro.

in $P$. falciparum and $P$. reichenowi. The distribution of coiled-coil regions in $P$. falciparum and $P$. reichenowi are similar where two coiled-coil regions are located after the signal peptide region and another one coiled-coil region present between 6-Cys region. Unlike P41 and P48/45 that have two 6-Cys domains, P230 has fourteen 6-Cys domains at most which mostly located after the signal peptide region. 


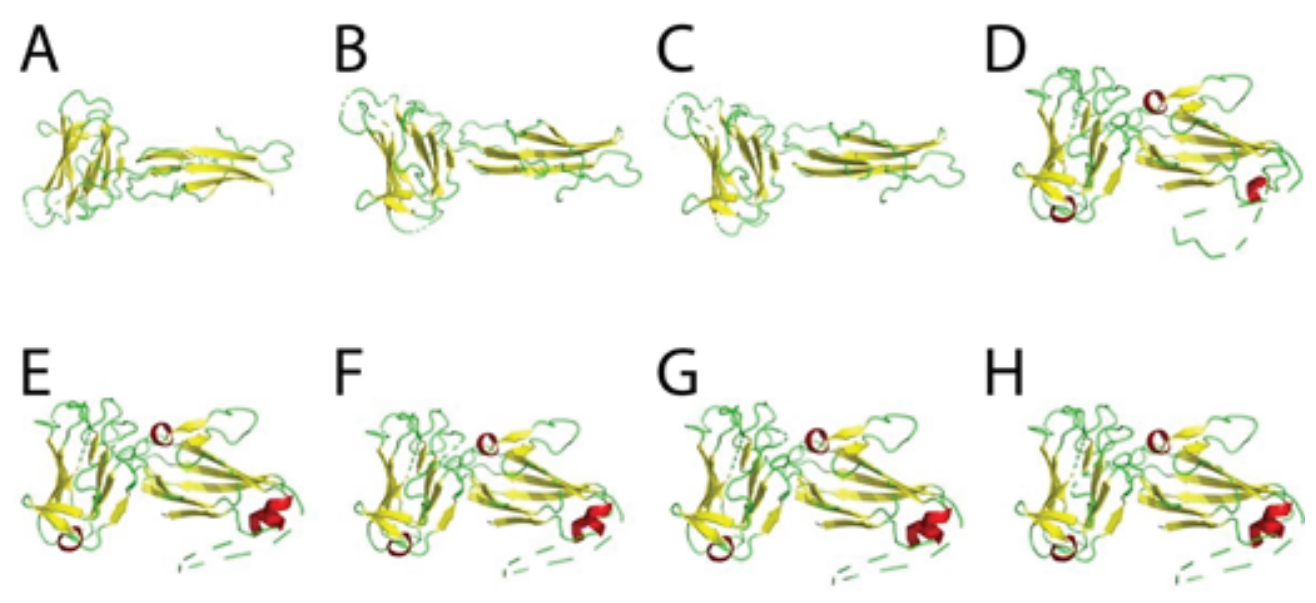

Figure 12: 3D protein structure of P230 protein predicted by using PHYRE2 and visualized by using PyMol version 2.3: (A) P. berghei, (B) P. chabaudi, (C) P. reichenowi, (D) P. falciparum, (E) P. vivax, (F) P. knowlesi, (G) P. ovale, and (H) P. malariae.

In 3D protein prediction, the significant differences in P230 protein structure were observed in human infecting and non-human infecting Plasmodium, where noninfectious Plasmodium does not have an alpha-helix structure compared to human infecting Plasmodium. The presence of alpha-helix structure in human-infecting Plasmodium may indicate that $\mathrm{P} 230$ protein in human infecting Plasmodium is more stable in a solution compared to non-human infecting Plasmodium [27].

\section{Conclusion}

Phylogenetic tree and time tree analysis revealed the unique evolutionary relationship in each of P41, P48/45, and P230 protein between human infecting and non-human infecting Plasmodium. Furthermore, the structural differences were observed in P230. Domain analysis also detected signal peptide, coiled-coil, and transmembrane region that could be used as a vaccine target. Thus, these results may support the development of a malaria vaccine using $\mathrm{P} 41, \mathrm{P} 48 / 45$, and $\mathrm{P} 230$ and may be useful for malaria control.

\section{Acknowledgment}

The authors would like to thanks the Indonesia International Institute (I3L) for their heartfelt support. Thanks also go to Giovanni Wijaya from Global Sevilla School for assisting this project during his summer internship in I3L. 


\section{Conflict of Interest}

The authors declare no conflict of interest in this research.

\section{Supporting Grants}

The research is funded by DRPM DIKTI funding with the scheme "Penelitian Dosen Pemula" in the 2019 funding year (Contract No: 48/AKM/MONOPNT/2019).

\section{References}

[1] WHO. (2019). WORLD MALARIA REPORT, 2018.

[2] Minister of Health Indonesia. (2018). Laporan Nasional Riskesdas 2018.

[3] Cowman, A. F., Healer, J., Marapana, D., \& Marsh, K. (2016). Malaria: Biology and Disease. Cell,167(3), 610-624. doi:10.1016/j.cell.2016.07.055

[4] Su, X., Lane, K. D., Xia, L., Sá, J. M., \& Wellems, T. E. (2019). Plasmodium Genomics and Genetics: New Insights into Malaria Pathogenesis, Drug Resistance, Epidemiology, and Evolution. Clinical Microbiology Reviews,32(4). doi:10.1128/cmr.00019-19

[5] Van der Pluijm RW, Imwong M, Chau NH, et al. (2019).Determinants of dihydroartemisinin-piperaquine treatment failure in Plasmodium falciparum malaria in Cambodia, Thailand, and Vietnam: a prospective clinical, pharmacological, and genetic study. Lancet Infect Dis 2019. doi: 10.1016/S1473-3099(19)30391-3.

[6] Bakhiet, A. M., Abdelraheem, M. H., Kheir, A., Omer, S., Gismelseed, et al. (2019). Evolution of Plasmodium falciparum drug resistance genes following artemisinin combination therapy in Sudan. Transactions of The Royal Society of Tropical Medicine and Hygiene. doi:10.1093/trstmh/trz059

[7] Arredondo, S. A., \& Kappe, S. H. (2017). The s48/45 six-cysteine proteins: Mediators of interaction throughout the Plasmodium life cycle. International Journal for Parasitology,47(7), 409-423. doi:10.1016/j.jpara.2016.10.002

[8] EBI. (n.d.). 6-Cysteine (6-Cys) domain. Retrieved August 5, 2019, from https://www. ebi.ac.uk/interpro/beta/entry/InterPro/IPR010884/

[9] Ahmed, M. A., Chu, K., \& Quan, F. (2018). The Plasmodium knowlesi Pk41 surface protein diversity, natural selection, subpopulation, and geographical clustering: A 6-cysteine protein family member. PeerJ,6. doi:10.7717/peerj.6141

[10] Srisutham, S., Saralamba, N., Sriprawat, K., Mayxay, M., Smithuis, F., Nosten, F.,... Imwong, M. (2018). Genetic diversity of three surface protein genes in Plasmodium 
malariae from three Asian countries. Malaria Journal,17(1). doi:10.1186/s12936-0182176-x

[11] Macdonald, N. J., Nguyen, V., Shimp, R., Reiter, K., Herrera, R., Burkhardt, M.,... Narum, D. L. (2016). Structural and Immunological Characterization of Recombinant 6Cysteine Domains of the plasmodium falciparum sexual Stage Protein Pfs230. Journal of Biological Chemistry,291(38), 19913-19922. doi:10.1074/jbc.m116.732305

[12] Aurrecoechea, C., Bertelli, J., Brunk, B. P., Dommer, J., Fischer, S., Gajria, B.,... Wang, H. (2009). PlasmoDB: A functional genomic database for malaria parasites. Nucleic Acids Research,37(Database). doi:10.1093/nar/gkn814

[13] Edgar, R. C. (2004). MUSCLE: Multiple sequence alignment with high accuracy and high throughput. Nucleic Acids Research,32(5), 1792-1797. doi:10.1093/nar/gkh340

[14] Hall, B. G. (2013). Building Phylogenetic Trees from Molecular Data with MEGA. Molecular Biology and Evolution,30(5), 1229-1235. doi:10.1093/molbev/mst012

[15] Jones, D. T., Taylor, W. R., \& Thornton, J. M. (1992). The rapid generation of mutation data matrices from protein sequences. Bioinformatics, 8(3), 275--282. doi: 10.1093/bioinformatics/8.3.275

[16] Mello, B. (2018). Estimating TimeTrees with MEGA and the TimeTree Resource. Molecular Biology and Evolution, 35(9), 2334--2342. doi: 10.1093/molbev/msy133

[17] Hedges, S. B., Marin, J., Suleski, M., Paymer, M., \& Kumar, S. (2015). Tree of life reveals clock-like speciation and diversification. Molecular Biology and Evolution, 32(4), 835--845. https://doi.org/10.1093/molbev/msv037

[18] Southan, C. (2000). InterPro (The Integrated Resource of Protein Domains and Functional Sites). Yeast,1(4), 327-334. doi:10.1002/1097-0061(200012)17:43.0.co;2-k

[19] Kelley, L. A., Mezulis, S., Yates, C. M., Wass, M. N., \& Sternberg, M. J. (2015). The Phyre2 web portal for protein modeling, prediction, and analysis. Nature Protocols,10(6), 845-858. doi:10.1038/nprot.2015.053

[20] Delano, W. L. (2002). Pymol: An open-source molecular graphics tool. CCP4 Newsletter on Protein Crystallography, 40, 82-92.

[21] Cornejo, O. E., \& Escalante, A. A. (2006). The origin and age of Plasmodium vivax. Trends in Parasitology,22(12), 558-563. doi:10.1016/j.pt.2006.09.007

[22] Prugnolle, F., Ollomo, B., Durand, P., Yalcindag, E., Arnathau, C., Elguero, E., ... Renaud, F. (2011). African monkeys are infected by Plasmodium falciparum nonhuman primate-specific strains. Proceedings of the National Academy of Sciences, 108(29), 11948--11953. doi: 10.1073/pnas.1109368108 
[23] Burdukiewicz, M., Sobczyk, P., Chilimoniuk, J., Gagat, P., \& Mackiewicz, P. (2018). Prediction of Signal Peptides in Proteins from Malaria Parasites. International Journal of Molecular Sciences,19(12), 3709. doi:10.3390/ijms19123709

[24] Céspedes, N., Connie S. N. Li Wai Suen, Koepfli, C., França, C. T., Felger, I., Nebie, I.,... Herrera, S. (2017). Natural immune response to Plasmodium vivax alpha-helical coiled-coil protein motifs and its association with the risk of P. vivax malaria. Plos One,12(6). doi:10.1371/journal.pone.0179863

[25] G.R. Coatney, W.E. Collins, M. Warren, et al. Plasmodium knowlesi. The primate malarias, U.S. Government Printing Office, Washington (1971), pp. 317-333

[26] Kovjazin, R., Volovitz, I., Daon, Y., Vider-Shalit, T., Azran, R., Tsaban, L., ... Louzoun, Y. (2011). Signal peptides and transmembrane regions are broadly immunogenic and have high CD8 T cell epitope densities: Implications for vaccine development. Molecular Immunology, 48(8), 1009--1018. doi: 10.1016/j.molimm.2011.01.006

[27] Cai, M., Arredondo, S., Clore, M., Miller, L., Takayama, Y., Macdonald, N.,... Aravind, L. (2012). Structure of the Plasmodium 6-cysteine s48/45 Domain. PNAS. doi:10.2210/pdb2loe/pdb 